\title{
Etude de programmation urbaine pour un projet résilient sur un site soumis au risque de submersion marine
}

\author{
Céline AZAÏS ${ }^{1}$, Antonin AMIOT ${ }^{2}$, Geoffrey CLAMOUR ${ }^{2}$, \\ Marie-Line CANOVILLE ${ }^{3}$, Pierre PEETERS ${ }^{4}$, Gaëlle SCHAUNER ${ }^{5}$
}

1. SETEC organisation, Immeuble Central Seine, 42/52 quai de la Rapée, CS 71230, 75583 Paris cedex 12, France.celine.azais@setec.com

2. Les Marneurs, 24 rue Léon Frot, 75011 Paris, France. contact@lesmarneurs.fr

3. Ville de Cherbourg-en-Cotentin, Direction urbanisme opérationnel et foncier 2 quai de Caligny, 50100 Cherbourg-en-Cotentin, France. marie-line.canoville@cherbourg.fr

4. SETEC hydratec, 16 Boulevard de l'Ecce Homo, 49100 Angers, France. pierre.peeters@setec.com

5. Cerema Normandie-Centre, DADT / GTPM, 10 Chemin de la Poudrière, CS 90245, 76121 Le Grand-Quevilly cedex, France.gaelle.schauner@cerema.fr

\section{Résumé :}

La ville de Cherbourg-en-Cotentin s'est historiquement développée à l'embouchure de la Divette, sur sa rive gauche, dans un paysage de lagunes et de marais mais à l'abri de la mer. Sa position stratégique à l'échelle de la Manche a fortement façonné son identité portuaire et maritime, effaçant progressivement ce territoire amphibie pour gagner des terres sur la mer (polder et endiguement).

Situé au fond de la petite rade de Cherbourg mais soumis à l'aléa de submersion marine, le site du quai Lawton-Collins présente un fort intérêt urbain, du fait de disponibilités spatiales et de sa localisation stratégique.

La problématique du rehaussement du niveau des océans et la prise en compte du risque de submersion marine sur ce secteur bas de Cherbourg-en-Cotentin a permis d'orienter cette reconquête maritime et littorale, en faisant du risque et de l'environnement, des outils d'aménagement et de programmation.

A travers l'analyse des atouts, des usages et des contraintes (en particulier la submersion), nous nous efforcerons de décrire l'approche méthodologique qui nous a permis d'aboutir, en co-construction, avec les parties prenantes, à la proposition d'une programmation urbaine résiliente permettant de concilier la gestion des risques submersion marine avec le développement économique et urbain du site et avec une attention particulière à sa forte valeur patrimoniale et culturelle.

\section{Mots-clés :}

Programmation urbaine, Submersion marine, Résilience, Environnement, Changement climatique, Géomorphologie littorale, Patrimoine, Culture du risque, Espace portuaire 


\section{Thème 7 - Risques côtiers}

\section{Le Quai Lawton-Collins, les enjeux de la reconquête du front de mer de Cherbourg-en-Cotentin}

1.1 L'artificialisation du trait de côte et la fermeture progressive de la rade

Le trait de côte de la rade de Cherbourg-en-Cotentin a fortement évolué depuis le XVIIe siècle. Sa transformation est étroitement liée à l'histoire portuaire et maritime de Cherbourg; de la création de l'arsenal à la poldérisation et à la réalisation du terre-plein des Mielles. En effet les plans du XVIIe siècle font état d'un faciès littoral très différent du paysage actuel : l'embouchure de la Divette et du Trottebec est libre et évolue en fonction des marées et des apports sédimentaires. Le littoral est composé d'un estran sableux (Mielles) qui se découvre à chaque marée basse. Le creusement du bassin du commerce, l'endiguement du lit de la Divette et du Trottebec ainsi que la construction de la digue du large au XVIIIe siècle sont les premières étapes de la fixation du trait de côte. S'ensuit une fermeture progressive de la rade par comblement des marais littoraux dans le secteur est (actuel quartier Val de Saire), par la canalisation et l'enfouissement de la Divette et du Trottebec et par la création de terre-plein en avancée sur la mer (terre-plein des Mielles).

L'artificialisation du trait de côte et l'enclavement du port a peu à peu éloigné la mer de la ville, favorisant l'oubli des dynamiques littorales. Ainsi sur les $20 \mathrm{~km}$ de trait de côte de la commune, seulement $10 \mathrm{~km}$ sont accessibles au public. Cependant on assiste aujourd'hui à l'émergence de nombreux projets de reconquête du front de mer et de valorisation de l'espace maritime et portuaire (plages vertes, aménagement du front de mer à Querqueville, Collignon, site de la Saline à Equerdreville-Hainneville).

\subsection{Des espaces publics soumis au risque d'inondation et de submersion marine}

Le quai Lawton Collins constitue le dernier endroit avec la jetée Chantereyne à offrir un contact direct avec l'eau et une vue dégagée sur l'horizon marin. Il y a un enjeu très fort de valorisation des espaces publics de ce quai. Ces espaces publics sont pour la plupart très minéraux et le végétal est relégué aux abords des voiries (alignements, rond-point) et aux collections botaniques (parc 19e et parc du muséum) qui rappelle que Cherbourg a été un port d'acclimatation pour les jardins du Roi. La majorité de ces espaces publics est située en zone inondable (par la Divette et le Trottebec) et en aléa submersion marine. Il $\mathrm{y}$ a un enjeu d'adaptation des grands espaces publics face au risque à l'échelle de Cherbourg-en-Cotentin. Le risque de submersion marine doit être caractérisé et géré à l'échelle de la petite rade afin de développer une approche cohérente de la résilience. L'analyse historique des épisodes de submersion marine enregistrés depuis la fin XVIIIe siècle révèle que l'avant-port et le quai L. Collins sont régulièrement inondés lors d'épisodes tempétueux (dépression, vents, grand coefficient de marée) mais également lors des grandes marées hivernales. Le dernier épisode datant du 10 mars 2008, correspond à la tempête Johanna. Le niveau a alors été mesuré à $+4.12 \mathrm{~m} \mathrm{NGF}$ au 


\section{XVI'mes Journées Nationales Génie Côtier - Génie Civil \\ Le Havre 2020}

marégraphe du port militaire et estimé à $+4.20 \mathrm{~m}$ NGF au niveau du bassin du Commerce (IMDC, 2016a).

La modélisation des aléas littoraux (IMDC, 2016b) sur le quai L. Collins met en évidence de nombreux secteurs en aléas forts (dans ce cas surtout lié aux vitesses d'écoulement). Le quai et les bâtiments, en l'état, ne sont pas adaptés au risque de submersion marine et en ce sens le principe de résilience doit être développé : à la fois dans l'aménagement des espaces publics, dans les bâtiments existants et à venir, mais également sur la prise de conscience du risque par les riverains et des futurs occupants et dans la prévention par la puissance publique.

\subsection{L'impact du changement climatique}

Les dernières projections du GIEC (2019), publiées en septembre 2019 dans un rapport spécial sur les océans et la cryosphère, donnent une plage probable d'élévation du niveau des océans de 0,29 m à 1,10 m d'ici 2100 par rapport à 1986-2005, selon trois trajectoires d'évolution du climat évaluées. Le littoral de la commune de Cherbourg-en-Cotentin ne serait alors pas épargné par cette élévation et doit dès lors s'adapter aux submersions plus fréquentes et à l'accentuation probable de l'érosion du trait de côte.

En prenant en compte la moyenne du scénario le plus impactant (RCP8.5), l'élévation du niveau moyen des mers serait de $+11 \mathrm{~cm}$ en $2026,+27 \mathrm{~cm}$ en 2050 et $+84 \mathrm{~cm}$ à 1 'horizon 2100 par rapport à la période 1986-2005. Avec ces hypothèses, le niveau marin pour un événement de type du 10 mars 2008 correspondant actuellement à une période de retour 40 ans (SHOM \& CETMEF, 2012), se verrait dépasser en moyenne une fois par an en 2070. En prenant les hypothèses plus pessimistes, ce niveau sera atteint près de deux fois par an d'ici 40 ans (2060) et sera dépassé à chaque marée de vive-eau en 2100.

\subsection{Les atouts urbains et les potentialités de valorisation}

Le quai Lawton Collins hérite d'un passé prestigieux, qui lui confère de nombreuses qualités architecturales et patrimoniales (gare maritime, ancienne gare transatlantique occupée par la Cité de la mer, entrepôts de chantiers navals, jetée, bords à quai, etc.). Par ailleurs sa position nord-sud offre aux promeneurs à la fois un panorama sur la ville historique (à l'ouest), un travelling sur le chenal ainsi qu'un dégagement sur l'horizon maritime au nord au-delà de la petite et de la grande rade. Son exposition nord-sud amène à se tourner vers l'intérieur des bassins plutôt que vers la mer, ce qui en fait une situation unique, surtout en période estivale, pour regarder le "coucher de soleil" sur les bassins de l'avant-port. Une autre qualité de ce site est de pouvoir offrir quelques points de contact avec l'eau, notamment au niveau de la cale des anciens chantiers navals "Bellot".

Au-delà de sa vocation d'espace public maritime, le quai reste très sollicité par l'activité portuaire, maritime et touristique : pêche (débarques, entretien, etc.), station de pilotage, douanes (garde-côtes), escales de paquebots de croisière, et de ferry transmanche. Dans ce sens ces activités doivent être en capacité d'évoluer et de s'adapter à l'élévation du 


\section{Thème 7 - Risques côtiers}

niveau de la mer mais également à l'augmentation des épisodes de submersion marine. Ainsi la présente étude proposera des attitudes d'adaptation des infrastructures associées à ces activités afin de pérenniser les vocations économiques, touristiques, culturelles, patrimoniales de ce site.

\section{Démarche itérative pour l'élaboration d'un programme d'aménagement résilient}

\subsection{Le risque comme générateur de projet urbain}

L'objectif de l'étude était de proposer un aménagement résilient co-construit dans le cadre d'un dialogue itératif entre programmation urbaine, enjeux environnementaux et projection du risque à l'horizon de 2100 , conformément au règlement du PPRL en cours d'élaboration.

Il devient un outil de conception et de programmation innovant et reproductible (dans la démarche). Ce parti-pris adopté par la commune tranche avec la manière dont elle s'est jusqu'alors urbanisée. Il s'agit à présent de s'adapter au changement climatique et à l'aléa submersion marine qui impactera fortement le secteur du quai Lawton-Collins à horizon 2100 , et non plus d'uniquement se protéger des contraintes naturelles (rades). Il s'agit aussi de réaffirmer le caractère maritime de Cherbourg-en-Cotentin en ouvrant la ville vers la mer. Ce changement de paradigme est doublé de l'objectif de faire évoluer progressivement la collectivité dans l'appréhension du risque et de la gestion de crise.

\subsection{La co-construction du projet urbain}

La commune a souhaité impliquer un large panel d'acteurs dans l'élaboration de la programmation du quai. Le schéma d'aménagement est de ce fait issu d'un long processus (16 mois) ponctué d'itérations avec les parties-prenantes. Cette co-construction était rendue d'autant plus nécessaire qu'un grand nombre d'acteurs interviennent sur ce quai, et pour certains d'entre eux, à plusieurs titres :

- La ville de Cherbourg-en-Cotentin (CEC), maître d 'ouvrage de l'étude

- La Communauté d'agglomération du Cotentin (CAC);

- Les partenaires institutionnels : Etat, Région notamment, l'Etablissement Public Foncier de Normandie et le CEREMA, intervenant au titre du CPIER ;

- Les services de l'Etat : l'UDAP (l'ancienne gare transatlantique est inscrite aux monuments historique), la DDTM (en charge du PPRL en cours d'élaboration), la sous-préfecture ;

- Les propriétaires fonciers : Etat, Ports de Normandie ;

- Les utilisateurs actuels : SPLA Cité de la Mer, entreprises et associations présentes sur le site, Ports de Normandie, concessionnaires des ports (CCI, SAS Port de Cherbourg), Etat ;

- Les utilisateurs projetés : le conseil local de la jeunesse ;

- Les usagers et la société civile : conseils de quartiers. 


\section{XVI'èmes Journées Nationales Génie Côtier - Génie Civil \\ Le Havre 2020}

Toutes les itérations ont également permis d'amorcer l'élaboration d'une culture commune du risque.

Outre les instances itératives traditionnelles représentées par les comités techniques et de pilotage, l'étude a été rythmée par quatre types de participation des parties-prenantes : les entretiens, la balade urbaine, le comité des usagers et les ateliers de co-construction.

\section{Conception du projet urbain}

\subsection{Garder la mémoire de l'ancien trait de côte : séquençage du quai}

Le parti pris qui a guidé la programmation du quai s'ancre dans son histoire maritime, que tous les acteurs locaux ont souhaité valoriser : l'ancien trait de côte revit et séquence le quai en trois zones figure 1.

- La séquence de la mer, située à l'extrémité nord du quai constitue un point de contact intense avec la mer. Ce ressenti est exacerbé par le dégagement de l'horizon et les perceptions sur le lointain. Les qualités de ce secteur sont renforcées par le rythme des départs et arrivées des bateaux et des paquebots. La morphologie affinée du quai au nord du pignon de l'ancienne gare transatlantique et la mise en scène du Redoutable invitent à une "plongée" vers l'univers subaquatique. Les intentions d'aménagements pressenties s'orientent vers une certaine sobriété, un reprofilage du bord à quai afin d'y accueillir des zones de repos / de contemplation en contact avec l'eau. Sur cette séquence le risque de submersion marine ne présente que très peu d'enjeux ; il s'agit de laisser la mer recouvrir le quai lors des grandes marées et des épisodes exceptionnels.

- La séquence de l'estran fait référence à l'ancien estran aujourd'hui remblayé. Cette portion du littoral correspond à la zone de marnage et est composée de nombreux écosystèmes "amphibies" d'une très grande diversité biologique. Située en partie centrale du quai, elle est structurée par l'ancienne gare transatlantique ainsi que l'ancienne jetée. L'estran aujourd'hui remblayé est aménagé de manière à gérer les eaux de ruissellement et le ressuyage des submersions marines. Ainsi un grand parc littoral composé de zones humides et de zones sèches permet d'accueillir une programmation multiple (activité saisonnière, évènements, équipements).

C'est également un secteur intégralement désimperméabilisé et planté d'essences de haut jet adaptées aux conditions climatiques locales (embruns, vents, etc.).

- La séquence de la dune correspond aux anciennes grèves détruites lors de la création des ouvrages maritimes et la création du quai. Aujourd'hui elle concentre de nombreuses activités portuaires et maritimes (pêche, douanes, pilotes) qui renforcent le caractère maritime du quai. Dans ce sens les orientations d'aménagement proposent de maintenir et valoriser ces activités (ateliers, aménagement du bord à quai, etc.) en combinant des espaces publics plantés (écosystèmes dunaires). Cette séquence joue un rôle prépondérant dans la réduction du risque de submersion marine. En effet le remblaiement et le 


\section{Thème 7 - Risques côtiers}

nivellement permettent l'implantation d'un quartier d'habitations et de services et protègent le secteur du Val-de-Saire très vulnérable aux aléas maritimes.
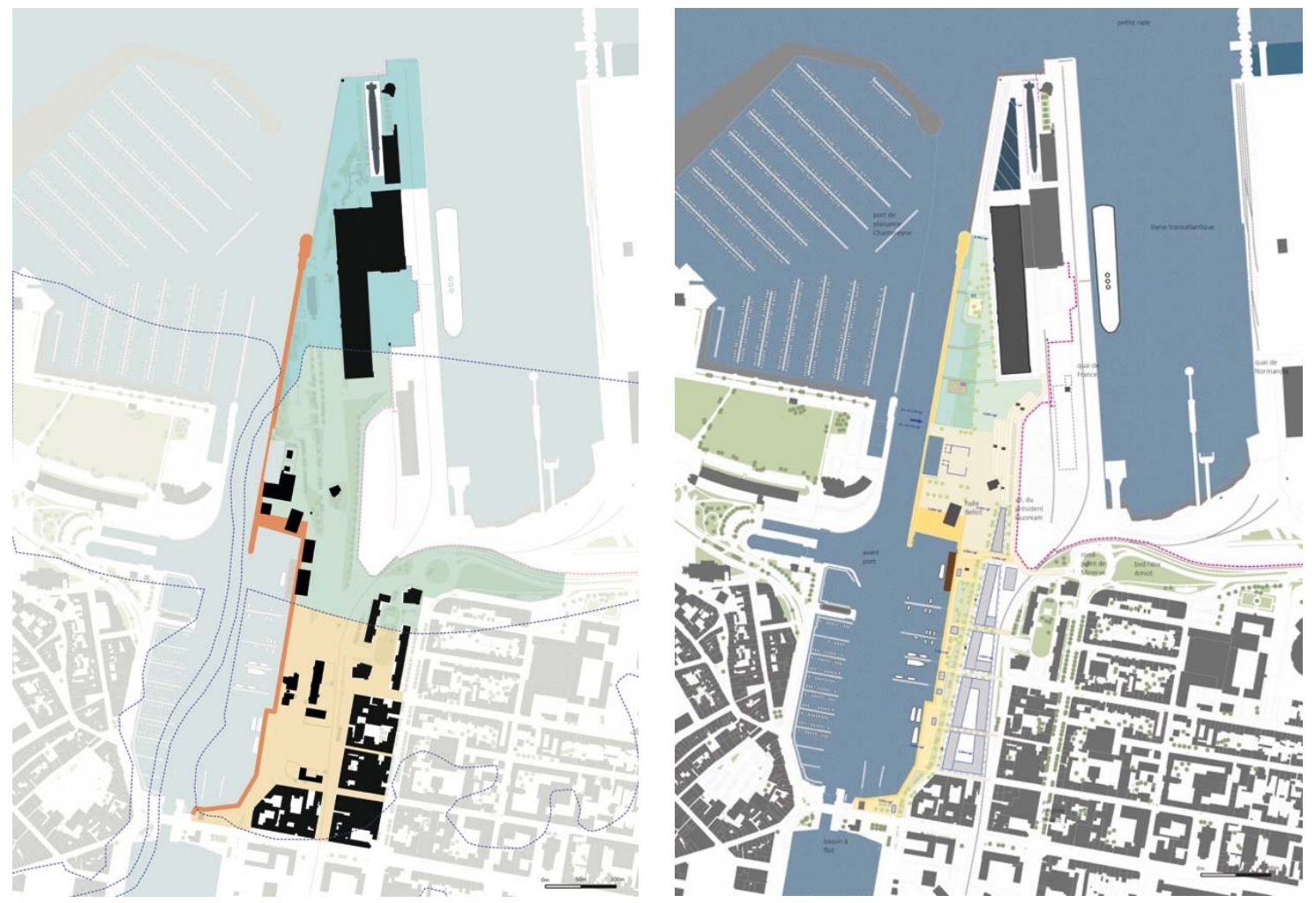

Figure 1. À gauche, cartographie du principe de séquençage retenu pour le rappel de l'histoire et de la géomorphologie du quai (en jaune la dune, en vert l'estran et en bleu la mer) / à droite, schéma d'aménagement urbain.

Si le nivellement du quai sur la partie voirie Lawton-Collins et la construction des RDC ne doivent pas déroger à la cote seuil du PPRN $(+5,25 \mathrm{mNGF})$, le projet ne prévoit pas de nivellement lié à une stratégie de protection sur le reste des autres secteurs, qui peuvent être inondables. En outre, les cotes réglementaires du PPRN n'intègrent qu'une augmentation du niveau marin de $60 \mathrm{~cm}$ sur la base de l'hypothèse "pessimiste" de l'ONERC (ONERC, 2010), publiée en 2010. Or depuis, les experts ont encore revu à la hausse leurs projections pour la fin du siècle, en 2014 puis plus récemment en 2019 (GIEC, 2014 ; GIEC, 2019). C'est pourquoi afin de proposer un aménagement résilient et après concertation avec les acteurs de la co-construction, la réflexion de la programmation urbaine se fera sur une hypothèse d'élévation du niveau marin de $+1 \mathrm{~m}$ à horizon 2100 , soit la cote $+5.50 \mathrm{mNGF}$ sur le secteur dunaire. Le programme prévoit dès lors un report du nivellement à $+5,50 \mathrm{mNGF}$ en cœur d'îlot afin de permettre une réduction partielle de la vulnérabilité de l'ouest du quartier du Val de Saire en cas d'événement exceptionnel. 


\section{XVI'èmes Journées Nationales Génie Côtier - Génie Civil \\ Le Havre 2020}

\subsection{Stratégies de résilience du bâti et des espaces publics}

Afin d'inscrire le développement du quai Lawton Collins dans une démarche d'innovation globale "pilote", nous avons donc souhaité approfondir autant que possible la question architecturale en prévoyant dès le stade de la conception la possibilité des bâtiments à s'adapter à différents niveaux de submersion, au-delà de la cote seuil du PPRN actuel. Pour cela, nous avons mis en place une approche dite "graduelle", qui articule en fonction des échelles de temps et d'aléa, différents types de dispositifs spatiaux, typologiques, constructifs et techniques.

Alors qu'en France la culture constructive privilégie la construction sur remblais ou sur pilotis, l'approche graduelle s'inspire des expériences d'Europe du Nord, comme Hambourg (Hafen City) ou Dodrecht et son concept de "multi-layered safety" (SOPHRONIDES et al., 2016), ainsi que des enseignements des rapports d'études du CEPRI sur l'adaptation du logement et de l'aménagement aux inondations (CEPRI, 2009 ; CEPRI, 2017). Plus précisément, l'approche graduelle propose d'articuler quatre stratégies complémentaires selon l'intensité de l'aléa et l'horizon temporel du changement climatique :

a) À court terme : Eviter

La stratégie d'aménagement et de construction doit répondre aux documents réglementaires actuellement en vigueur. Il s'agit donc de suivre la stratégie "classique" de remblaiement des emprises constructibles jusqu'à la côte $+5,25 \mathrm{mNGF}$ en suivant le plan guide d'aménagement, sachant que cette côte sera très probablement obsolète à moyen/long terme.

b) À moyen terme : Résister

Il est donc nécessaire d'anticiper ces changements, en proposant dès le stade de la conception des bâtiments des mesures conservatoires permettant d'envisager le comportement résilient des bâtiments au-delà de la côte de référence $+5,25 \mathrm{mNGF}$.

Dans le cas de submersions marines, qui sont des évènements ponctuels, progressifs (ne génèrent pas de courant d'écoulement forts) et limités dans le temps (le temps d'une marée haute) de nombreuses solutions architecturales existent pour rendre les bâtiments plus résistants : mise en place de dispositifs (portes, fenêtres...) antisubmersion, choix de matériaux hydrofuges en façade ou résistants à la corrosion et au sel, éviter l'isolation par l'extérieur en rez-de-chaussée.

c) À long terme : Céder

L'étude propose également de pousser la prospective vers des scénarios aujourd'hui considérés comme très pessimistes ou encore non considérés, où lors de marées hautes exceptionnelles, la hauteur de la lame d'eau pourrait provoquer une pression trop forte sur la construction, et contraindrait à laisser entrer l'eau dans le bâtiment. Il s'agit ici de mettre en œuvre une stratégie "à la vénitienne", grâce à des solutions constructives permettant la pénétration de l'eau et le redémarrage rapide et peu coûteux de l'activité 


\section{Thème 7 - Risques côtiers}

du bâtiment après l'inondation. La mise en œuvre de planchers refuges, de matériaux hydrofuges, de systèmes constructifs et de réseaux adaptés ou de systèmes de ventilation naturelle (etc.) sont des solutions à mettre en œuvre de manière coordonnée pour y parvenir.

d) De 2020 à 2100, et après : Préserver

Enfin, il y aurait peu de sens de concevoir des bâtiments résilients si ces derniers, par leur bilan carbone contribuaient par effet ricochet à nourrir la dynamique de submersion. Une stratégie de résilience du bâtiment ne peut donc être complète si elle ne prend pas en compte le problème à la racine, en intégrant les notions de sobriété, d'énergie grise, et de bilan carbone : minimiser la matière, choix de matériaux bio et géo-sourcés, isolants d'origine végétale, conception bio-climatique, etc.

\subsection{Une méthodologie innovante}

Afin de préciser conceptuellement et techniquement les quatre stratégies de l'approche graduelle, l'étude met en place un "référentiel des solutions résilientes" compilant un grand nombre de mise en œuvre possible, qui sont ensuite traduits spatialement suivant des coupes architecturales génériques, figure 2.

Enfin, l'étude apporte également une approche pré-opérationnelle et économique en développant la faisabilité d'un ilot résilient témoin, à partir duquel sont estimés les coûts induits de mise en œuvre des différents dispositifs résilients.

Ces deux derniers points représentent une innovation en soi, étant donné le manque de référentiel unifié et d'estimations économiques dans la littérature existante sur l'aspect résilience architecturale.

\section{Conclusion}

La démarche mise en place pour l'élaboration du projet du quai Lawton-Collins a une portée globale, à l'échelle de l'ensemble de la commune. Elle incite la ville à s'interroger sur une stratégie globale de résilience de ses espaces publics inondables, mais aussi dans le cadre de ses opérations d'aménagement, et de réhabilitation d'équipements publics. Cette démarche a également permis la sensibilisation de l'ensemble des acteurs, y compris les services techniques, à tous les enjeux liés au risque de submersion marine. 


\section{XVİ̀mes Journées Nationales Génie Côtier - Génie Civil \\ Le Havre 2020}

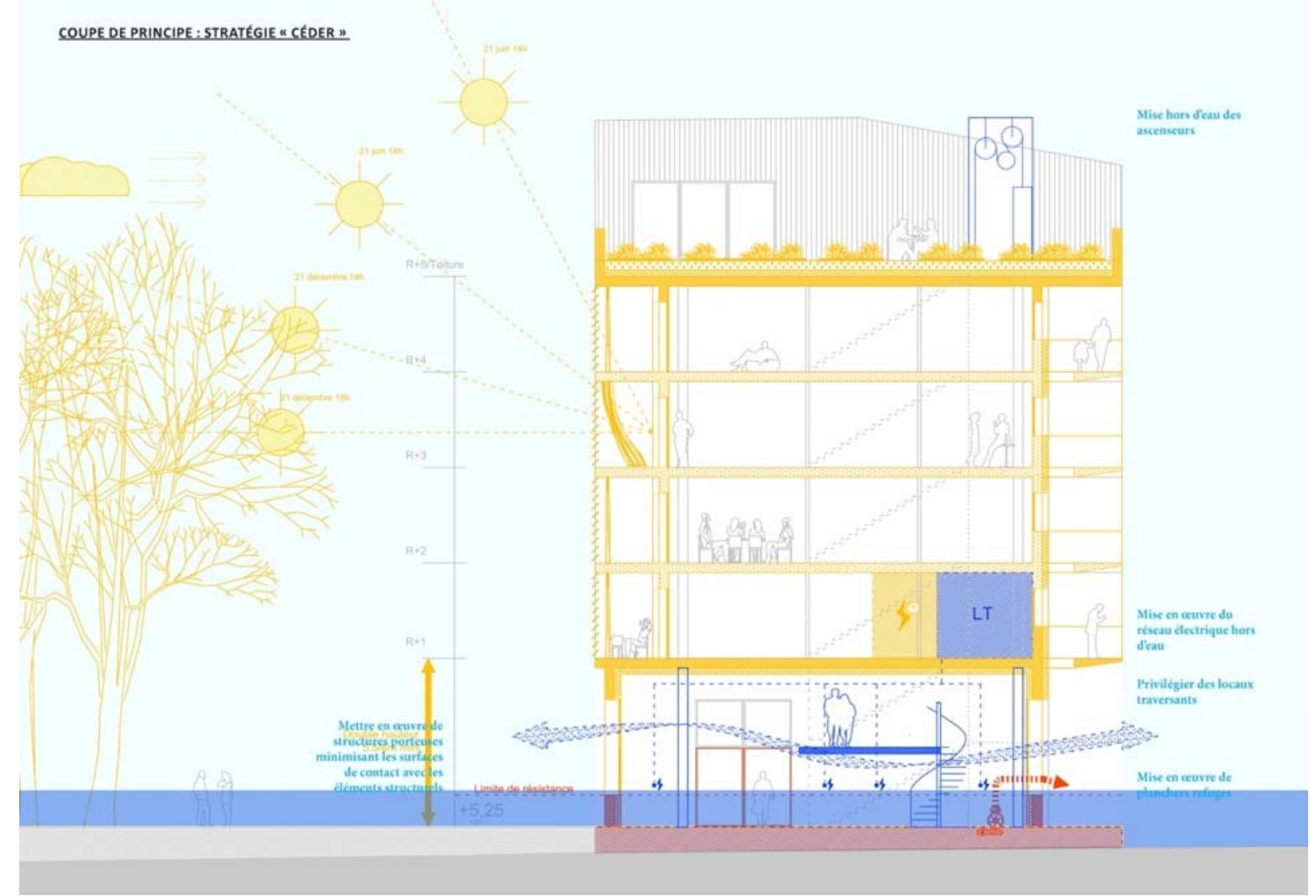

Figure 2. Coupe des principes architecturaux et techniques de résilience de stratégies ERCP (Eviter / Résister / Céder / Préserver).

\section{Références bibliographiques}

CEPRI (2009). Un logement "zéro dommage" face au risque d'inondation est-il possible ? Rapport, $56 \mathrm{p}$.

CEPRI (2017). La réalisation d'opérations d'aménagement résilientes aux inondations : qui gagne quoi ? Rapport, $72 \mathrm{p}$.

GIEC (2014). Climate change 2013. Rapport d'évaluation du Groupe d'experts intergouvernemental sur l'évolution du climat (V). 1552 p.

GIEC (2019). Ocean and cryosphere in a changing climate - Chapter 4: Sea level rise and implications for low-lying islands, coasts and communities. Rapport spécial du GIEC, $126 \mathrm{p}$.

IMDC (2016a). Plan de Prévention des Risques Naturels Multirisques : submersion marine, inondations par débordement de cours d'eau et chutes de blocs de la région cherbourgeoise - Phase 2 Caractérisation des aléas : Evénements de référence au large, Rapport, $122 \mathrm{p}$.

IMDC (2016b). Plan de Prévention des Risques Naturels Multirisques : submersion marine, inondations par débordement de cours d'eau et chutes de blocs de la région cherbourgeoise - Phase 2 Caractérisation des aléas : Modélisation de submersion, de l'érosion du trait de côte et cartographie des aléas, Rapport, 138 p. 
Thème 7 - Risques côtiers

ONERC (2010). Synthèse - Prise en compte de l'élévation du niveau de la mer en vue de l'estimation des impacts du changement climatique et des mesures d'adaptation possibles. 6 p. https://www.ecologique-solidaire.gouv.fr/sites/default/files/synth\%20niveau\%20mer.pdf

SHOM, CETMEF (2012). Statistiques des niveaux marins extrêmes des côtes de France (Manche et Atlantique), Édition SHOM.

SOPHRONIDES P., STEENBRUGGEN J., SCHOLTEN H., GIAOUTZI M. (2016). Geodesign the multi-layered water safety. Research In Urbanism Series, 4(1), pp 113-138. https://doi.org/10.7480/rius.4.825 